\title{
Optimization of Multi-Wavelength Interdigital Dielectrometry Instrumentation and Algorithms
}

\author{
A. V. Mamishev, B. C. Lesieutre and M. Zahn \\ High Voltage Research Laboratory, Lab. for Electromagnetic and Electronic Systems, \\ Massachusetts Institute of Technology, Cambridge, MA
}

\begin{abstract}
Interdigital frequency-wavelength dielectrometry can be used to measure the dielectric permittivity and conductivity of insulating materials. The complex dielectric permittivity is directly related to other material properties, such as moisture content, temperature, concentration of impurities and additives, density, aging status, etc. The analysis of spatial and temporal variations of these properties lends valuable insights into physical phenomena which take place in electrical equipment, provides instrumentation for system monitoring and diagnostics, and can be used for optimization of design and performance of electrical apparatus. The optimization of various aspects of this technology is described in this paper. Improvement of performance is achieved through variation of geometrical design, materials, manufacturing processes, and electronic circuitry. Accumulated effects of non-ideal geometry of the experimental setup and the sensor itself are accounted for through empirical measurements, calibration, and use of finite-element calculations. Three distinct operating modes are developed: floating voltage with grounded backplane, floating voltage with guarded backplane, and short circuit current. Measurements reveal that the interfacial contact quality has a strong influence on the sensor's response. Gain/phase measurements over the frequency range $5 \mathrm{mHz}$ to $10 \mathrm{kHz}$ agree well with theoretical calculations on the interfacial contact quality. Full-frequency measurements for several liquid and solid dielectrics are shown to have a good match with theoretical predictions.
\end{abstract}

\section{INTRODUCTION.}

$\mathrm{D}$ EVELOPMENT of interdigital $\omega-k$ (frequency-wavenumber) dielectrometry and application of this technology for characterization of insulating materials has been continuing for over two decades $[1-6]$. The work reported here builds heavily on the results of previous research $[2,7,9]$. One of the most active areas of interest is the application of interdigital dielectrometry for measurement of moisture diffusion processes in transformer pressboard [9-11]. Some of the first practical implementations of the multi-wavelength sensors exhibited noticeable differences between the theoretical predictions and the measurements of interelectrode impedances. When the estimation problem of material characterization is being solved, relatively small discrepancies between theory and measurements can lead to large errors. Modifications to the measurement techniques and algorithms which allow better diagnostics of performance of the parameter estimation routines are described here.

\section{GENERAL THEORETICAL BACKGROUND}

\subsection{FORWARD AND INVERSE PROBLEMS}

A conceptual schematic of $\omega-k$ dielectrometry is presented in Figure 1. Periodic variation of electric potential along the surface in the $y$ direction produces an exponentially decaying pattern of electric fields penetrating into the medium in the $z$ direction. The forward problem for this study can be defined as determination of the field distribution and the interelectrode admittance matrix given the geometry, material properties, and external excitations. The inverse problem usually involves determination of either material properties or associated geometry, or both, given the imposed excitations and experimental values associated with the admittance matrix of the system.

From the electroquasistatic field point of view, the electric scalar potential of the field excited by the driven electrodes is a solution to 
Laplace's equation. At any constant $z$ position, if the sensor is many wavelengths wide, far from the ends the field is periodic in the $y$ direction and assumed uniform in the $x$ direction. The scalar potential can be written as an infinite series of sinusoidal Fourier modes of fundamental spatial wavelength $\lambda$ that decays away in the $z$ direction $[2,7,9]$

$$
\phi=\sum_{n=0}^{\infty} \phi_{n} \exp \left[-k_{n} z\right]\left(A_{n} \sin k_{n} y+B_{n} \cos k_{n} y\right)
$$

where $k_{n}=2 \pi n / \lambda$ is the wavenumber of each mode.

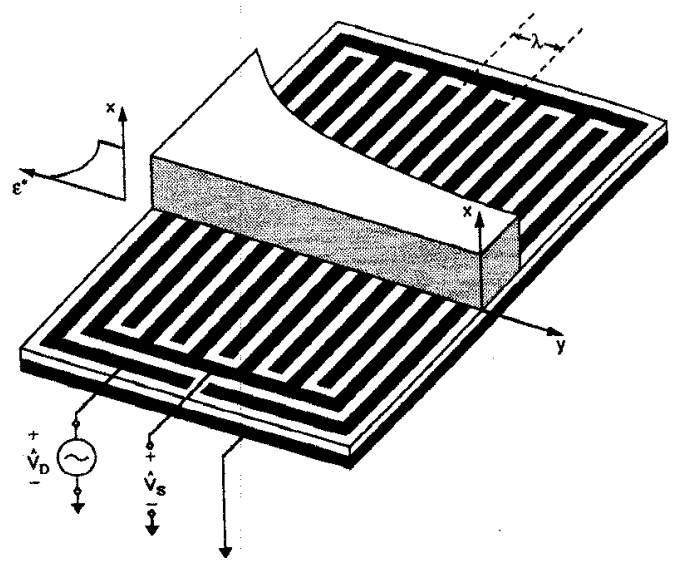

Figure 1. Imposed $\omega-k$ dielectrometry [2].

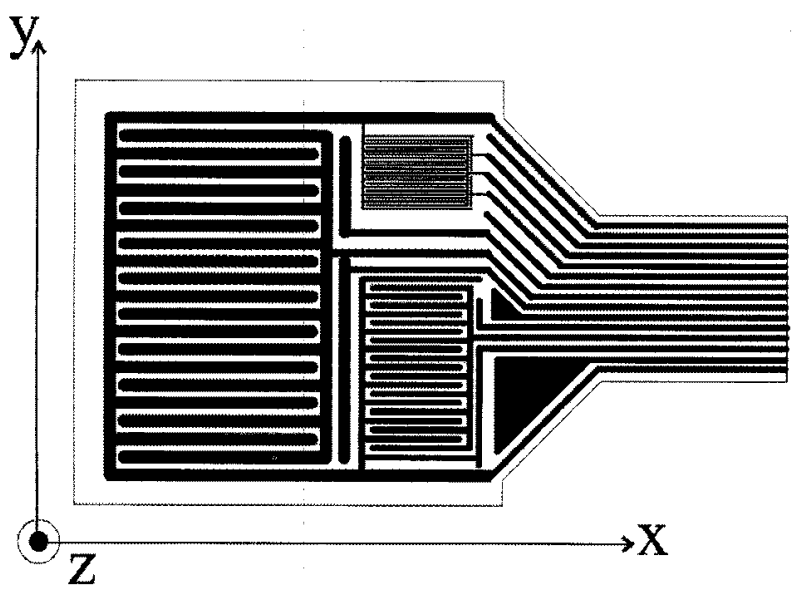

Figure 2. Earlier design of the three-wavelength interdigital sensor [7].

It is possible to solve for the field distribution associated with a given excitation and geometry in closed-form using a continuum model. This model has been described extensively in [2,9]. The model uses the concept of a complex surface capacitance density $\widehat{C}_{n}$, which relates at a planar surface of constant $z$ to the potential $\hat{\phi}_{n}$ at that surface for every Fourier mode $n$ in the following way

$$
\widehat{C}_{n}=\frac{\varepsilon^{*} \widehat{E}_{z n}}{\widehat{\phi}_{n}}
$$

where

$$
\varepsilon^{*}=\varepsilon-\mathrm{i} \frac{\sigma}{\omega}
$$

is the complex permittivity with $\varepsilon$ the material dielectric permittivity and $\sigma$ the ohmic conductivity. Then, knowing $\widehat{C}_{n}$ at the electrode surface will let us calculate the terminal current from the potential distribu- tion at that surface, which is essentially equivalent to forward-solving Equation (1).

Although this continuum model offers some advantages inherent to the closed-form solution, it is also to limited to geometries in which the electrodes are assumed to have zero thickness, and the dielectric mâterials are stratified into parallel homogeneous layers of uniform thickness and properties. Driven by the need to account for numerous nonideal geometry effects arising in experiments, this study based forwardproblem solution on finite-element (FE) techniques, using the commercial electromagnetic (EM) simulation software Maxwell ${ }^{\mathrm{TM}}$ by Ansoft Corporation [12].

In both cases, whether the continuum model or FE approach is used, the inverse problem is usually solved iteratively, by guessing at material or geometry properties, solving the forward problem, and comparing the simulation with the experimental data. Uniqueness of solution of the inverse problem has been postulated for certain conditions specific to a given geometry, such as distinct relaxation times for different layers of materials or non-dispersive frequency properties $[7,13,14]$. The cases addressed in this study admit unique solutions.

\subsection{DIELECTRIC PROPERTIES}

In most cases, the dielectric response of insulating materials is frequency dependent. Several mechanisms are associated with this phenomenon and represent a field of study in itself [15]. In addition to bulk dispersion, when $\varepsilon$ and $\sigma$ in (3) are frequency dependent, surface effects, such as surface conductivity, formation of an electrical double layer, and interfacial charge accumulation take place and affect the outcome of experiments $[7,16]$. Such phenomena are not the subject of this particular study, and their influence has been minimized by appropriate choice of test specimens with homogeneous and almost non-dispersive complex permittivity in the frequency range of interest $(5 \mathrm{mHz}$ to $10 \mathrm{kHz})$.

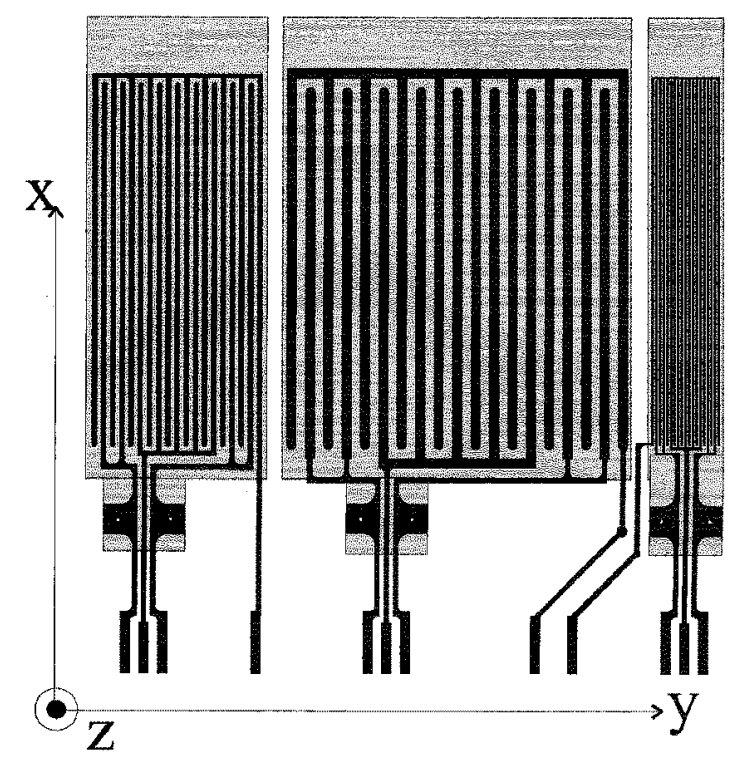

Figure 3. Latest design of the three-wavelength interdigital sensor. 


\section{THREE-WAVELENGTH SENSOR}

\subsection{GENERAL DESIGN}

\subsubsection{PREVIOUS DESIGN}

The earlier design of the three-wavelength sensor has been described thoroughly in $[7,10]$ and other publications. The planar view of this design is shown in Figure 2. This sensor consists of three sets of copper electrodes on a flexible polyimide Kapton ${ }^{\mathrm{TM}}$. (Du Pont) substrate with wavelengths of 1,2.5 and $5 \mathrm{~mm}$. The sensing electrodes are shielded in the plane of the sensor by guard electrodes driven by a buffer stage in the interface circuit, and the guard electrodes are shielded by ground electrodes [9]. A thin $(5 \mu \mathrm{m})$ layer of Parylene ${ }^{\mathrm{TM}}$ covers the sensor on both sides to prevent moisture absorption by the hydrophilic Kapton substrate. In general, presence of moisture in the volume of Kapton causes significant variations of its complex dielectric permittivity. A grounded backplane deposited on the bottom side of the substrate provides shielding from external perturbations, defines ground potential, and confines the electric field to the upper half-space.

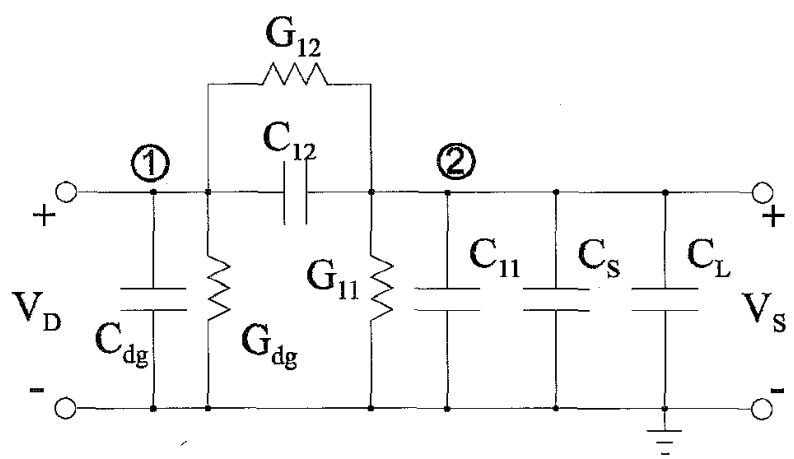

Figure 4. Floating voltage measurement with an impedance divider.

The idealized model for which the closed form expression for the electric field exists requires the electrodes to be an infinitely long and wide array of zero height metal microstrips placed on the surface of the insulating substrate. The most important contributors to the discrepancies between the theoretical model and the real measurement data are the thickness of the copper electrodes ( 35 or $17.5 \mu \mathrm{m}$ for different sensor designs), the finite length and finite number of the fingers [17], the capacitance of the leads of the electrodes to ground and to each other, the metalization ratio (described as the ratio of the area covered with copper to the total area of the sensor), and the fluid (typically air or liquid dielectric) trapped between the sensor's surface and the surface of the tested specimen [18].

\subsubsection{NEW DESIGN}

The latest design structure of the three-wavelength interdigital sensor used in this investigation is shown in Figure 3. The major features, the number of wavelengths and their spatial periodicity of $1,2.5$ and $5 \mathrm{~mm}$ are kept the same as in the previous Kapton sensor. The substrate material was changed from Kapton ${ }^{\mathrm{TM}}$ to Teflon ${ }^{\mathrm{TM}}$ (Du Pont). The superior hydrophobic characteristics of Teflon necessitated this choice, as it eliminated the need of the moisture barrier coating, although Kapton outperforms Teflon in its thermal and mechanical properties. The recent
Table 1. Differences between the first (Kapton substrate) and new (Teflon substrate) design of the three-wavelength interdigital sensor.

\begin{tabular}{|l|l|l|}
\hline \hline & \multicolumn{1}{|c|}{ Previous design } & \multicolumn{1}{|c|}{ New design } \\
\hline $\begin{array}{l}\text { Substrate material } \\
\text { Coisture barrier }\end{array}$ & $\begin{array}{l}\text { Kapton }\left(\varepsilon_{r}=3.05\right) \\
\text { Parylene }(5 \mu \mathrm{m} \\
\text { thick) }\end{array}$ & Teflon $\left(\varepsilon_{r}=2.1\right)$ \\
\hline $\begin{array}{l}\text { Thickness of } \\
\text { substrate }\end{array}$ & $127 \mu \mathrm{m}$ & $254 \mu \mathrm{m}$ \\
\hline Metallization ratio & 0.5 & 0.5 \\
\hline Measured quantity & Floating voltage & $\begin{array}{l}\text { Floating voltage or } \\
\text { short-circuit current }\end{array}$ \\
\hline $\begin{array}{l}\text { Mode of operation, } \\
\lambda=1,2.5,5 \mathrm{~mm}\end{array}$ & $\begin{array}{l}\text { All wavelengths } \\
\text { simultaneously }\end{array}$ & $\begin{array}{l}\text { Either simultaneous or } \\
\text { separate }\end{array}$ \\
\hline $\begin{array}{l}\text { Nominal meander } \\
\text { length (m) }\end{array}$ & $0.3 / 0.3 / 0.6$ & $0.5 / 0.5 / 0.5$ \\
\hline $\begin{array}{l}\text { Thickness of } \\
\text { electrodes }\end{array}$ & $35 \mu \mathrm{m}$ or $17 \mu \mathrm{m}$. & $14 \mu \mathrm{m}$. \\
\hline $\begin{array}{l}\text { Number of sensing } \\
\text { electrode fingers per } \\
\text { wavelength }\end{array}$ & 10 & 5 \\
\hline Connection & Flat cable & Coaxial cable \\
\hline Backplane & Common & Separate \\
\hline \hline
\end{tabular}

introduction by Du Pont of Kapton-F, which consists of a Kapton core with Teflon-like coating may make it possible to manufacture a sensor on a substrate which combines the advantages of both materials.

Guard fingers are introduced on each side of the active sensing area to make the sensor look 'infinite' in the $y$ direction. Table 1 lists the important parameters for the Kapton and Teflon substrate sensors. The nominal meander length represents the total length of the meandering dielectric path between the sensing and the driven electrodes, which is equal to twice the total length of the sensor electrode fingers.

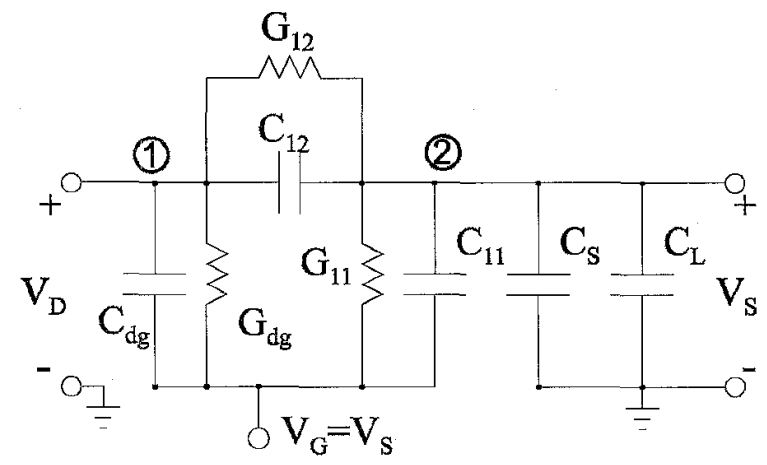

Figure 5. Floating voltage mode measurement of gain, phase, $C_{12}$ and $G_{12}$ with actively driven backplane voltage equal to the sensing voltage.

\subsection{MANUFACTURING AND CLEANING OF THE NEW TEFLON SENSOR}

A thin layer of copper with bonding strength of 0.3 to $0.6 \mathrm{~kg} / \mathrm{cm}^{2}$ can be attached to the surface of the Teflon substrate by electroplating. Alternatively, lamination can be used, however, lamination of Teflon is less effective than that of most other insulating polymers. Standard etching techniques allow a copper electrode line edge resolution of $12.5 \mu \mathrm{m}$, which is sufficient for the spatial wavelengths on the order of millimeters required in this design. A one-time cleaning procedure specific for 
this particular manufacturing process is required after the sensors are manufactured in order to remove the chemical agent responsible for adhesion of copper to Teflon from the areas where the copper was etched away. After several iterations, the following cleaning procedure was followed:

1. Sensors are rinsed with acetone and methanol and dried at $50^{\circ} \mathrm{C}$ for $15 \mathrm{~min}$ to remove dust, fingerprints, etc.

2. In the meantime, regular bleach solution is heated to $65^{\circ} \mathrm{C}$ and degassed at maximum setting and average power in an ultrasonic cleaner for $10 \mathrm{~min}$. The degassing is needed to reduce the mechanical impact of ultrasonic cleaning on the copper electrodes.

3. The power setting of the ultrasonic cleaner is reduced to the minimum, and degassing is turned off. Sensors are placed inside the ultrasonic tank for 1 to $2 \mathrm{~min}$. Naturally, the maximum possible time depends on a particular model of the ultrasonic cleaner. In our case, the ULTRAsonik 104x by NEY was used. When the sensors' surface turns from brown (color of the bonding agent) to white, the ultrasonic cleaning is complete.

4. Sensors are removed from the cleaning tank and flushed with running warm water, then rinsed with acetone, immediately followed by rinsing with methanol. Each consecutive rinsing must be done before the sensor dries off, otherwise, a residue remains deposited on the surface.

5. Sensors are baked in the oven at $50^{\circ} \mathrm{C}$ for $1 \mathrm{~h}$.

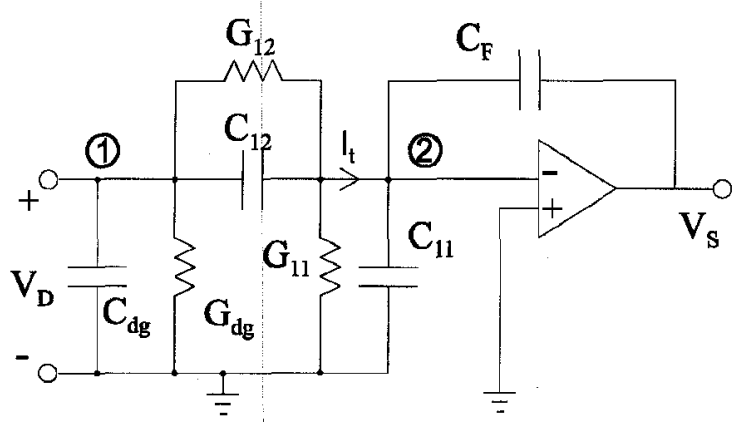

Figure 6. Short-circuit mode measurement of sensor electrode current $I_{t}, C_{12}$ and $G_{12}$ by current integration.

Subsequent routine cleaning of the sensor can be done with regular solvents, such as acetone and methanol, with little or no heating required afterwards.

\subsection{OPTIMIZATION OF VARIOUS FEATURES}

\subsubsection{INTERFACE CIRCUITRY}

Under any type of sensor geometric arrangement and electronic control, estimation of dielectric properties of materials is performed by determining measurable quantities defined by an equivalent lumpedelement circuit and relating them to the complex permittivity. The measurement of profiles of other physical variables $[9,19]$, such as the moisture distribution, requires a calibration mapping relating the complex permittivity to the physical variable. Appropriate choice of measurable quantities may significantly simplify this task. Possible variations of the measurement setup compared in this Section are (a) floating voltage measurement with backplane held at ground potential, $(\mathrm{b})$ floating voltage measurement with backplane and possibly guard fingers held at guard potential, (c) short circuit current measurement with backplane and possibly guard fingers held at virtual ground potential, and (d) combinations of the above three approaches.
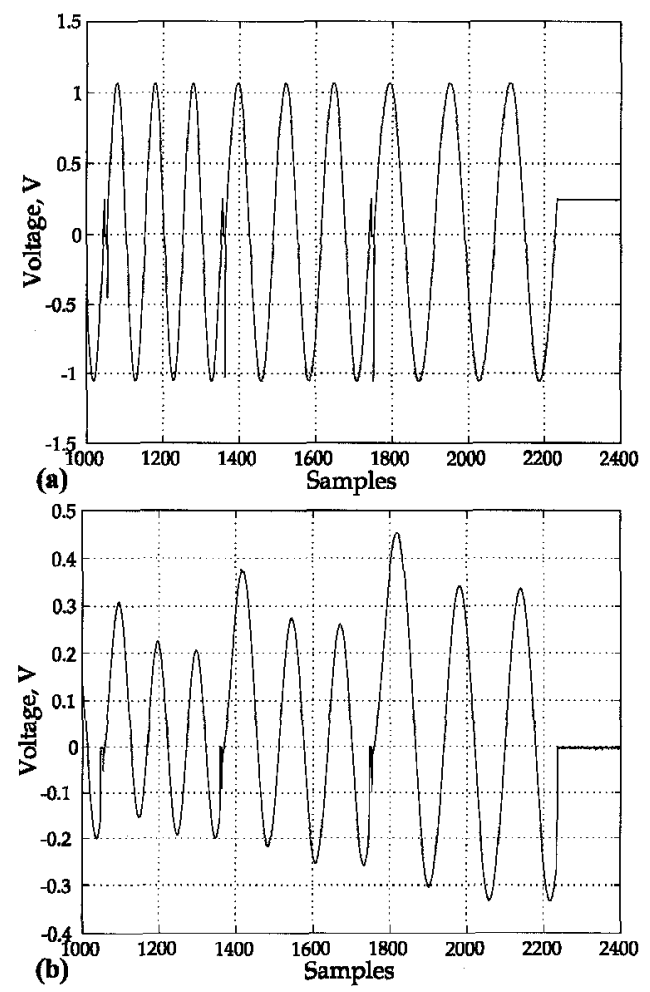

Figure 7. (a) Driven electrode time-domain sinusoid, (b) sensing electrode time-domain response.

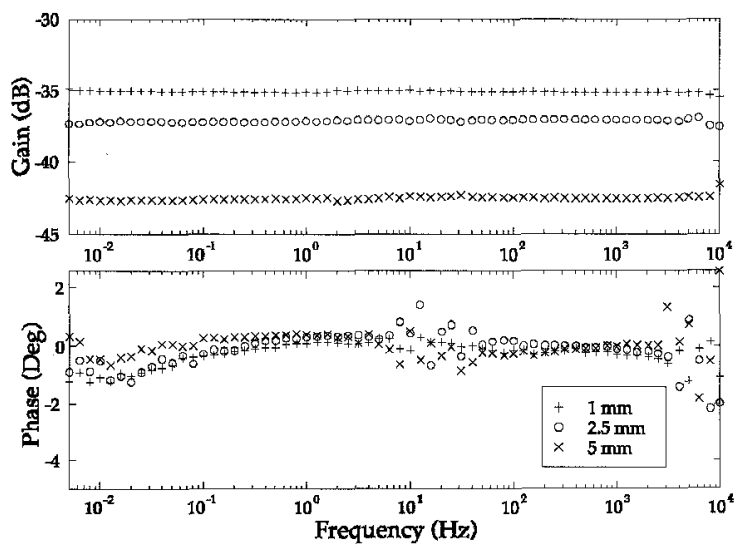

Figure 8. Kapton sensor - full frequency sweep in air. Values of load capacitances are given in Table 2. Phase is close to zero and the gain is flat over the entire frequency range since the interelectrode admittance is purely imaginary and frequency independent.

The following discussion gives comparisons of their features.

\section{Floating voltage with ground plane}

The circuit schematic for this measurement mode is presented in Figure 4. Node 1 corresponds to the driven electrode, and node 2 to the sensing electrode. Basically, an impedance divider is formed by the sensor's equivalent lumped elements and by the load capacitance $C_{L}$. Strictly speaking, the load capacitance is not necessary, since the admittance to the ground plane is connected in parallel, but it is useful to minimize the effects of the unknown stray capacitance $C_{S}$, and to optimize the amplitude of the complex gain. The complex gain between the 


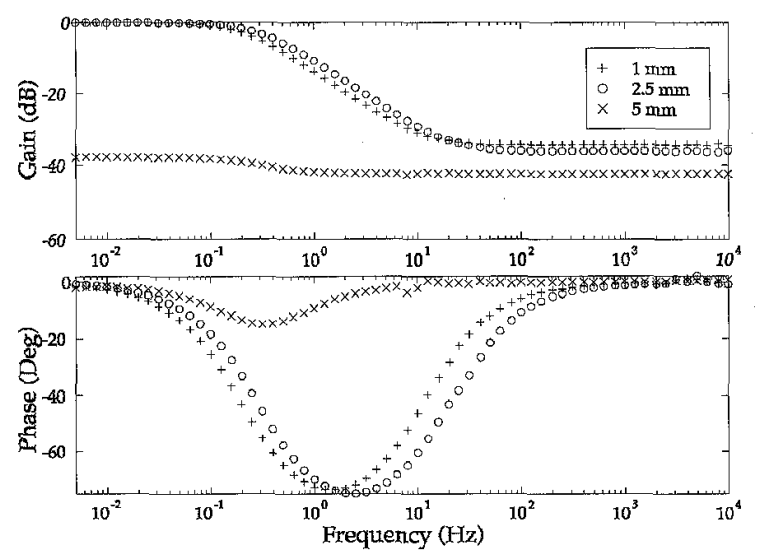

Figure 9. Kapton sensor - full frequency sweep in air. Two resistors of 9.8 G Reach are separately connected in parallel with $1 \mathrm{~mm}$ and $2.5 \mathrm{~mm}$. wavelengths. Ideally, the response of the $5 \mathrm{~mm}$ wavelength should remain unchanged from that shown in Figure 8. However, the phase changes to $-15^{\circ}$ and the gain increases by $\sim 4 \mathrm{~dB}$ at low frequencies due to crosscoupling between channels.

driven and the sensing voltage at frequency $\omega$ is given by

$$
\begin{aligned}
\widehat{G} & =\frac{\widehat{V}_{S}}{\widehat{V}_{D}} \\
& =\frac{G_{12}+\mathrm{i} \omega C_{12}}{G_{12}+G_{11}+\mathrm{i} \omega\left(C_{12}+C_{11}\right)+\mathrm{i} \omega\left(C_{L}+C_{S}\right)}
\end{aligned}
$$

where $\widehat{V}_{S}$ is the phasor voltage of the sensing electrode, and $\widehat{V}_{D}$ is the phasor voltage of the driven electrode. It should be remembered that elements $G_{12}, G_{11}, C_{12}$, and $C_{11}$ are frequency-dependent, as they represent an equivalent network of distributed elements that differs topologically from the actual sensor. $C_{S}$ represents the combined effects of the stray capacitance of the sensor leads, gate of the operational amplifier, fringing fields to the surrounding objects, etc. The sum $\left(C_{11}+C_{L}\right)$ is usually one or two orders of magnitude larger than $C_{S}$.

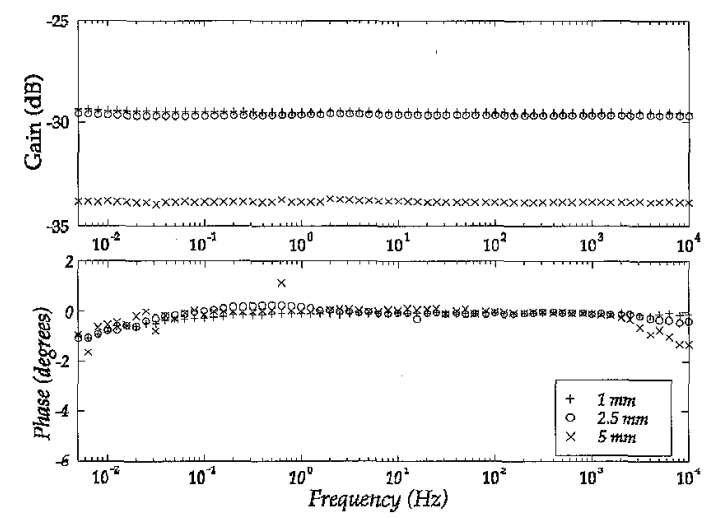

Figure 10. Clean Teflon sensor - full frequency sweep in air. Values of load capacitances are given in Table 2 . The slight decrease in phase at low frequencies sometimes takes place due to residual contamination and existence of conduction paths in the electronic circuitry, but can be ignored for most measurements as long as it stays within the noise level.

A disadvantage of this measurement approach is that the complex gain values cannot be converted in a straightforward way to the equivalent network element values. One complex equation can be solved for at most two real unknowns, while Equation (4) has four unknowns $\left(G_{12}\right.$,
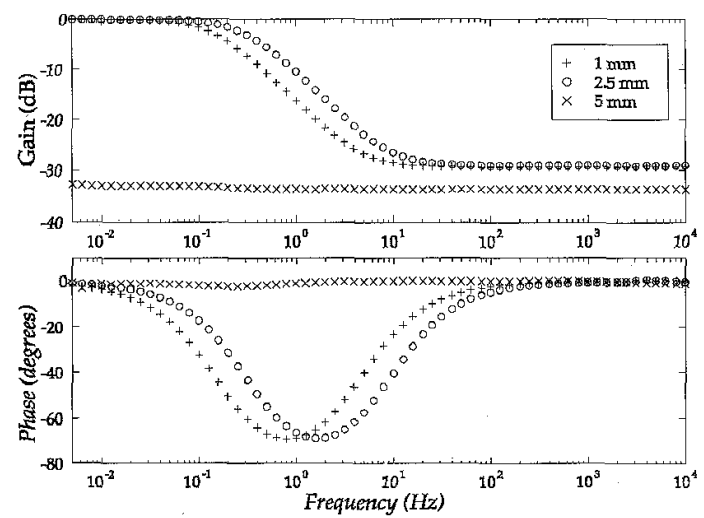

Figure 11. Teflon sensor - a full frequency sweep in air similar to that for Kapton sensor, presented in Figure 9. Two resistors of $9.8 \mathrm{G} \Omega$ each are separately connected in parallel with $1 \mathrm{~mm}$ and $2.5 \mathrm{~mm}$ wavelengths. The response of the $5 \mathrm{~mm}$ wavelength is practically unaffected by the other two.

$G_{11}, C_{12}$, and $\left.C_{11}\right)$ when the complex gain is known from measurements.

A similar argument applies to the high frequency limit, where equivalent conductances $G_{12}, G_{11}$ can be ignored compared to the imaginary part of all admittances. In this case, only one equation with two unknowns is available

$$
G=\frac{V_{S}}{V_{D}}=\frac{C_{12}}{C_{12}+C_{11}+C_{L}^{\prime}}
$$

where the gain is now real and $C_{S}$ was incorporated into $C_{L}^{\prime}=C_{L}+$ $C_{S}$.

Although the number of equations is not sufficient to solve directly for equivalent capacitances and conductances, the solution can be found by iterative solution of the electric potential distribution either by a continuum model or by finite-element techniques. That is, the solution to the inverse problem can still be found, by employing two additional implicit functional dependencies, $G_{12}(\varepsilon, \sigma, \omega)$ and $C_{12}(\varepsilon, \sigma, \omega)$ found by theory. This approach is not necessarily reliable, due to a high sensitivity to the error and possible existence of multiple solutions.

One way to simplify the relationship between measurable quantities and equivalent admittance values is to drive the backplane at the same potential as the sensing voltage. A detailed description of this approach follows.

\section{Floating voltage with guard plane}

When the potential equal to that of a floating sensing electrode is applied to the backplane, $V_{G}=V_{S}$, as shown in Figure 5 , the equivalent circuit elements $G_{11}$ and $C_{11}$ no longer affect the complex gain of the impedance divider, so that (4) reduces to

$$
\widehat{G}=\frac{\widehat{V}_{S}}{\widehat{V}_{D}}=\frac{G_{12}+\mathrm{i} \omega C_{12}}{G_{12}+\mathrm{i} \omega C_{12}+\mathrm{i} \omega\left(C_{L}+C_{S}\right)}
$$

This simplification allows the sequence of the inverse problem solution process to be changed. The equivalent admittance between the driven and sensing electrodes can now be found easily by solving a system of two linear equations with two unknowns. Relating the value of the complex interelectrode admittance to the $\varepsilon^{*}$ of the tested material can be achieved in several ways, which are discussed in the appropriate following sections. 


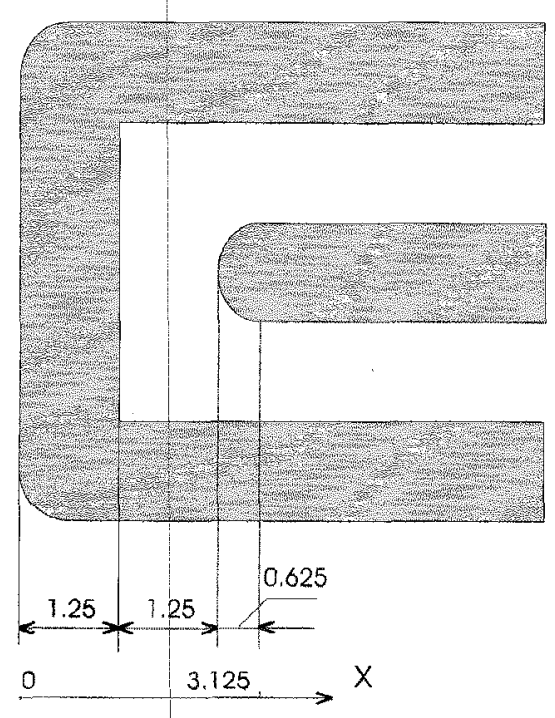

Figure 12. An enlarged image of the end area of the individual finger of the $5.0 \mathrm{~mm}$ wavelength sensing electrode [23].

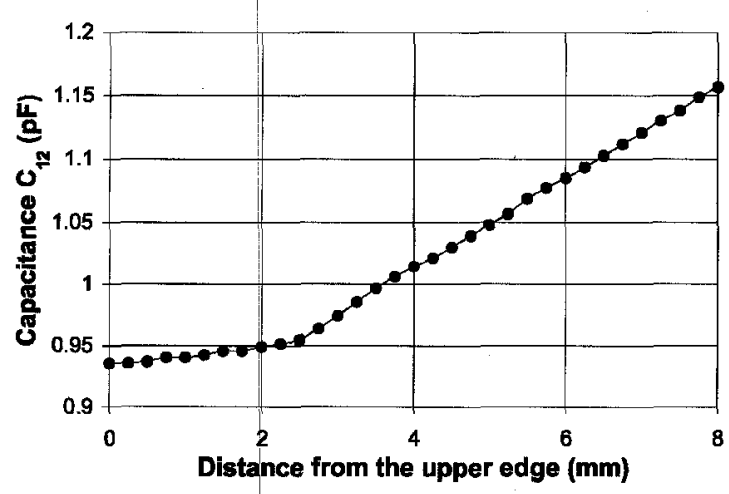

Figure 13. Sensor's response to the linear movement of the Lexan ${ }^{\mathrm{TM}}$ slab [23].

There are several consequences of the change of the measurement approach which affect the design of the sensor. Since the backplane has to be driven at the guard voltage, and this voltage is different for each wavelength, it is no longer possible to have a common backplane, as was implemented in the previous design. This is why the new design has three separate backplanes, shown in gray in Figure 3. Also, any grounded electrodes, such as a shielding case, must be placed sufficiently far from the sensing electrode, so that the elimination of $C_{11}$ in (6) is valid. In addition, separation of the driven electrode into three independent units is desirable if precise measurements are needed by avoiding cross-coupling with other wavelengths. In this case, only one wavelength at a time performs active measurements, and all other electrodes are held at the guard potential of the active sensing electrode.

\section{Short circuit current with virtual ground}

A measurement setup [20] which combines advantages of both guard plane and ground plane approaches is presented in Figure 6. Measurement of the current $I_{t}$ and knowledge of the voltage $V_{D}$ provides the sought information about the properties of the circuit elements $G_{12}$ and

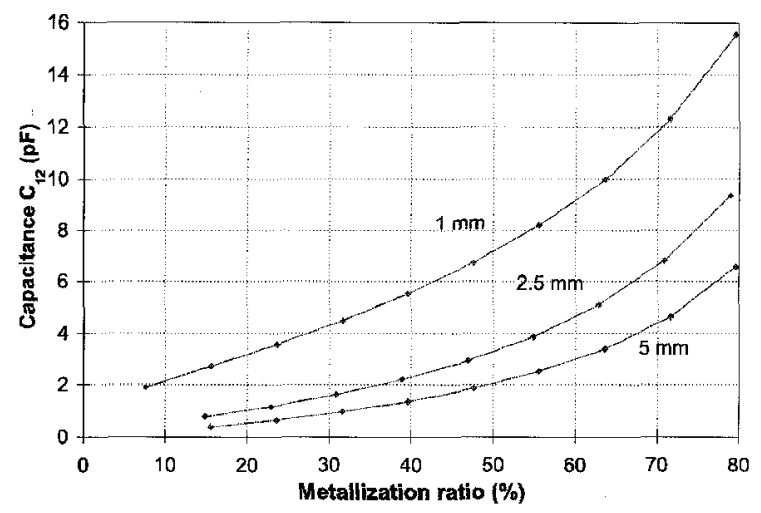

Figure 14. Variation of capacitance $C_{12}$ per meter length with the width of the electrodes, while the spatial wavelength stays constant for each case.

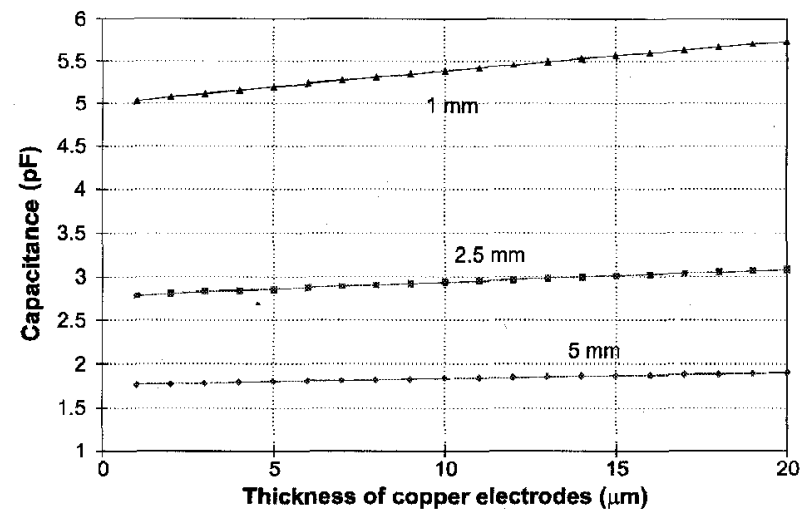

Figure 15. Dependence of the parasitic capacitance on the thickness of electrodes for all three wavelengths.

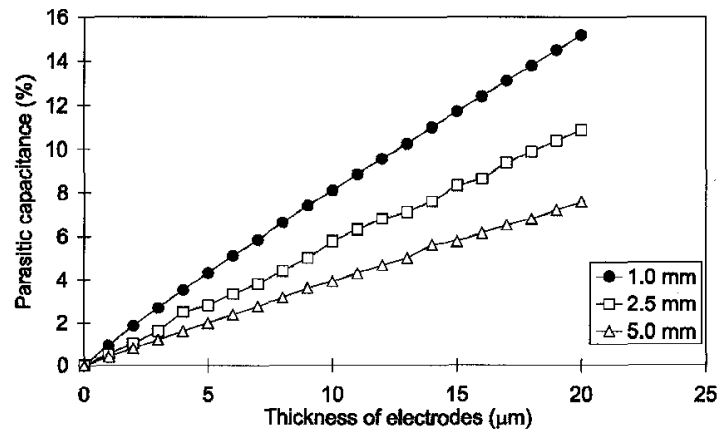

Figure 16. Percentt error of sensor output introduced by parasitic capacitance due to finite height electrodes as compared to zero thickness electrode analysis [23]

$C_{12}$

$$
\begin{array}{r}
G_{12}(\omega)=\Re\left(\frac{\widehat{I}_{t}}{\widehat{V}_{D}}\right) \\
C_{12}(\omega)=\frac{1}{\omega} \mathfrak{I}\left(\frac{\widehat{I}_{t}}{\widehat{V}_{D}}\right)
\end{array}
$$

In this case, the backplane, as well as all surrounding electrodes are always held at the ground potential. At very low frequencies, the voltage build-up of the op-amp input may exceed the saturation voltage, in which case the value of the feedback loop capacitor $C_{F}$ needs to be 
increased. Automatic switching of the feedback capacitor to avoid low frequency saturation is implemented electronically.

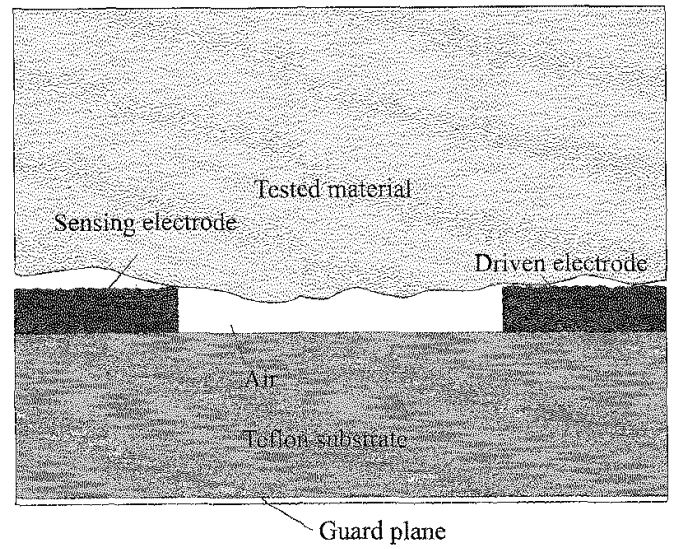

Figure 17. Air layer trapped between the drive and the sensing electrodes during measurements with solid materials due to electrode height, surface roughness, and surface non-flatness. For all calculations the electrode height is $14 \mu \mathrm{m}$, the Teflon substrate has thickness $254 \mu \mathrm{m}$ and $\varepsilon_{r}=$ 2.1 , and the tested material has infinite height.

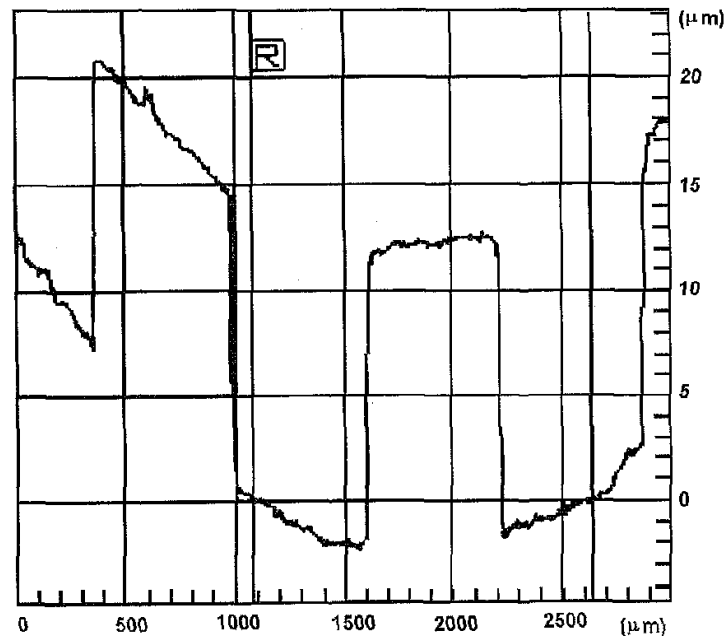

Figure 18. Profilometer scan of $2.5 \mathrm{~mm}$ wavelength. The $\sim 625 \mu \mathrm{m}$ wide electrodes are located approximately at coordinates 300 to $900 \mu \mathrm{m}$ and 1600 to $2200 \mu \mathrm{m}$. Variation of baseline due to slight tilt of supporting platform is software-corrected in the region 1100 to $2600 \mu \mathrm{m}$.

\section{Time-domain measurement data}

Illustrative time-domain measurement data are shown in Figure 7 during a three data point interval of the frequency sweep at approximately $0.03,0.04$, and $0.05 \mathrm{~Hz}$ on the $5 \mathrm{~mm}$ wavelength electrodes immersed in corn oil. Data are taken at a sampling frequency of $10 \mathrm{~Hz}$. One can see that the switching transient is of practically zero duration for the driven voltage in Figure 7(a). The voltage on the sensing electrode cannot be measured directly, because the measurement itself would distort the signal in such a high impedance application. The guard voltage, equal to the sensing electrode voltage, is shown in Figure $7(b)$. The sensing electrode voltage can be viewed as a combination of particular and homogeneous solutions of a differential equation approximately describing the distributed circuit of the sensor. Sinusoidal steady-state is
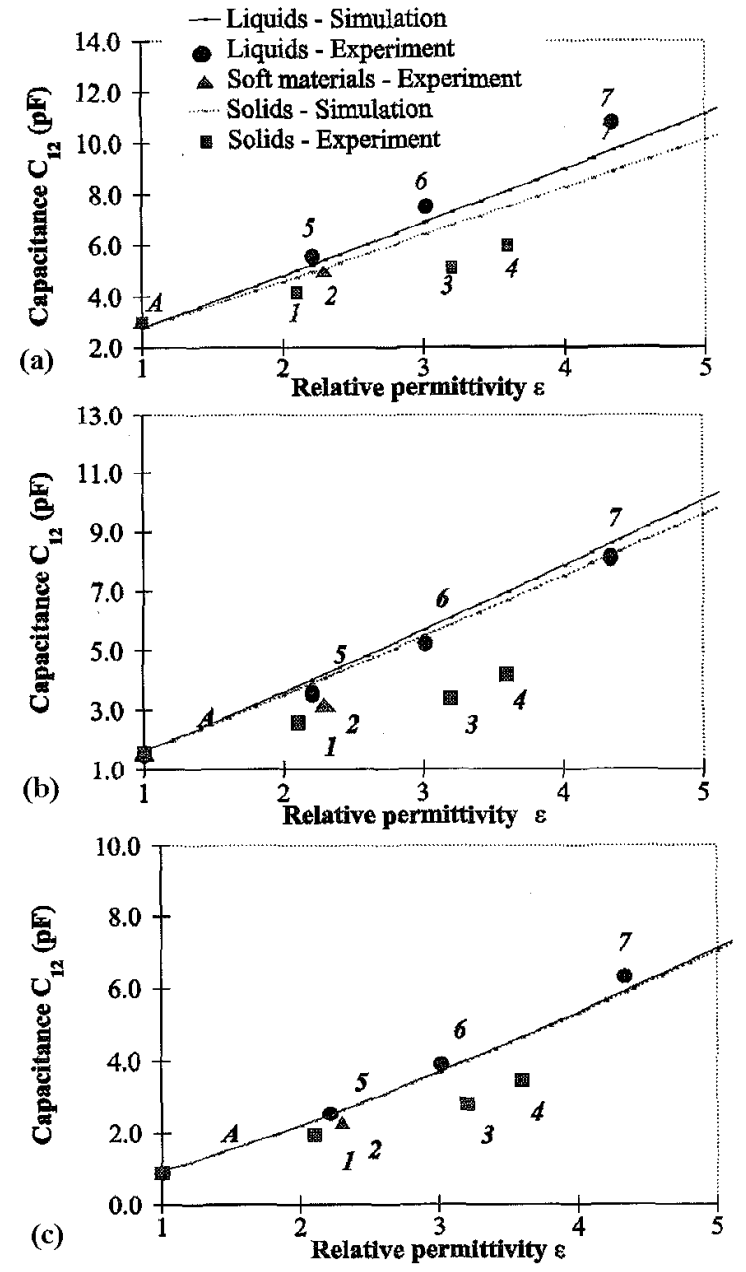

Figure 19. Measured and calculated interelectrode capacitance $C_{12}$ for air, liquid, and solid dielectrics for each of the sensor's three wavelengths. Legend A: air, 1: Teflon, 2: Polyethylene, 3: Lexan, 4: Delrin, 5:Transformer oil, 6: corn oil, 7: castor oil. (a) $\lambda=1 \mathrm{~mm}$, (b) $\lambda=2.5 \mathrm{~mm}$, (c) $\lambda=$ $5 \mathrm{~mm}$.

nearly achieved by the end of the third cycle, which is used for measurement of gain and phase of a capacitive divider. The residual dc offset is accounted for in associated controller firmware by subtracting an estimated value of residual dc offset from the signal during the third cycle of the measurement.

\subsubsection{CROSS COUPLING.}

In the initial design, shown in Figure 2, the driven electrode was shared between the three sensing electrodes of different spatial periodicity. Consequently, the excitation of all wavelengths and measurement of their response was done simultaneously. Due to close placement and the two-dimensional in-plane (as opposed to the three-dimensional) shielding of the leads, the voltage induced on the sensing electrode (and associated shielding circuitry) of one wavelength affects the response of the others. This effect was significantly reduced in the new design. Shown in Figure 8 is a frequency sweep of the Kapton sensor in air. This sweep should be compared to a similar one, shown in Figure 9 , where $9.8 \mathrm{G} \Omega$ resistors were connected in parallel with each 2.5 and $1 \mathrm{~mm}$ wavelength sensor electrode. Since no changes were made on the 


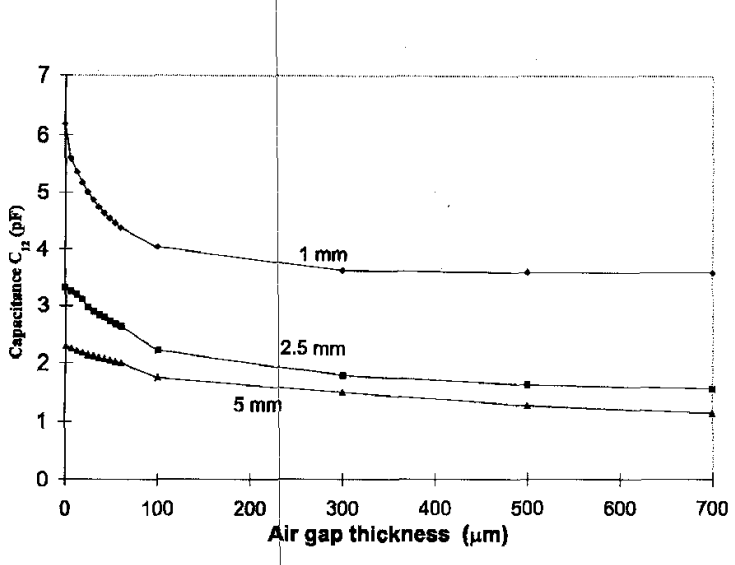

Figure 20. Simulation of the air gap for all three wavelengths, assuming the test dielectric has $\varepsilon_{r}=2.1$.

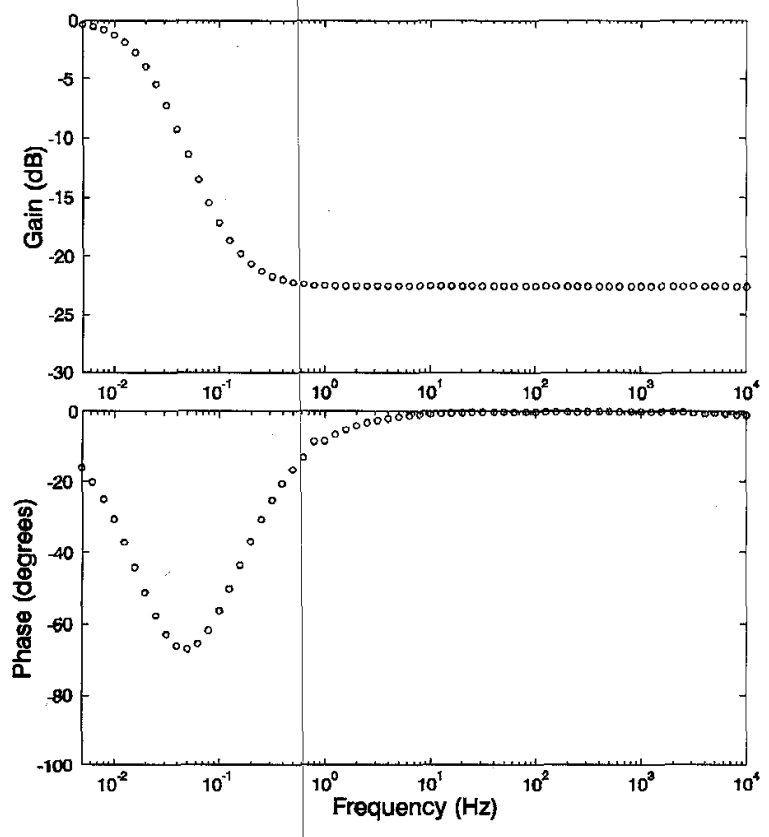

Figure 21. Measurement of corn oil with a floating voltage with guard plane method, $5 \mathrm{~mm}$ wavelength, load capacitance $47.4 \mathrm{pF}$.

$5 \mathrm{~mm}$ wavelength channel, the response for this wavelength should remain the same as in Figure 8 at all frequencies. However, both gain and phase of the $5 \mathrm{~mm}$ wavelength channel undergo transition at the midfrequency range, caused by cross-coupling from the other two wavelengths revealed by the sensor electrode loading by $9.8 \mathrm{G} \Omega$ resistors.

An equivalent pair of frequency sweeps for the new sensor is shown in Figures 10 and 11. In this case, the effect of cross-coupling is practically negligible. In many cases with the Teflon sensor, especially when properties of materials do not have a strong variation in space, crosscoupling effects are negligible, and the measurements can be performed simultaneously on all wavelengths. Cases where the variation of the complex permittivity in space is strong, sometimes require independent, non-simultaneous measurements.

\subsubsection{REDUCTION OF FRINGING FIELD EFFECTS}

Two types of end discontinuities in Figure 1 must be considered: the finite number of fingers along the $y$ axis, and the finite length of the elec-
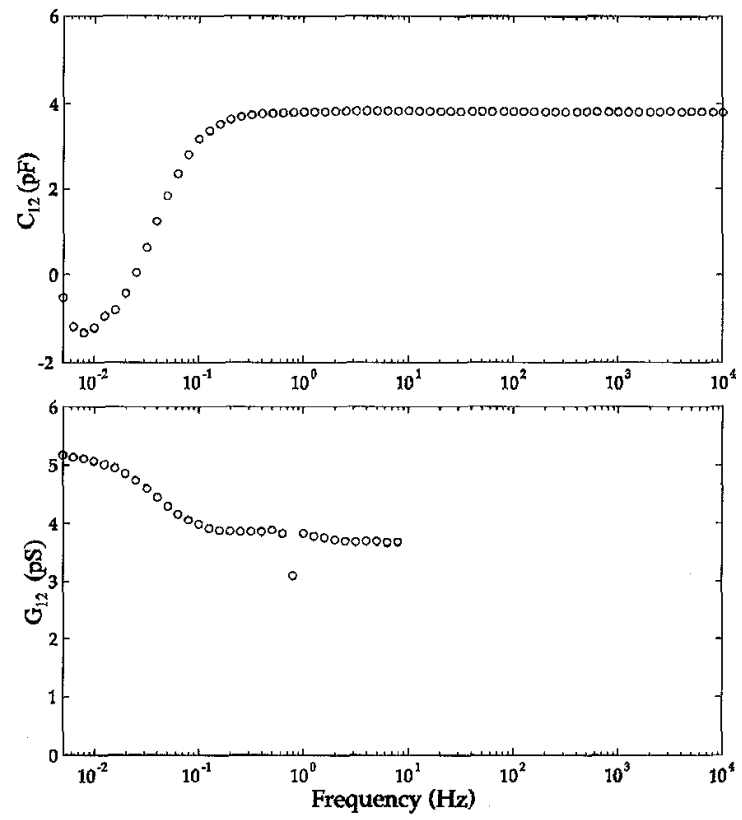

Figure 22. Interelectrode admittance for the corn oil experiment with $5 \mathrm{~mm}$ wavelength.

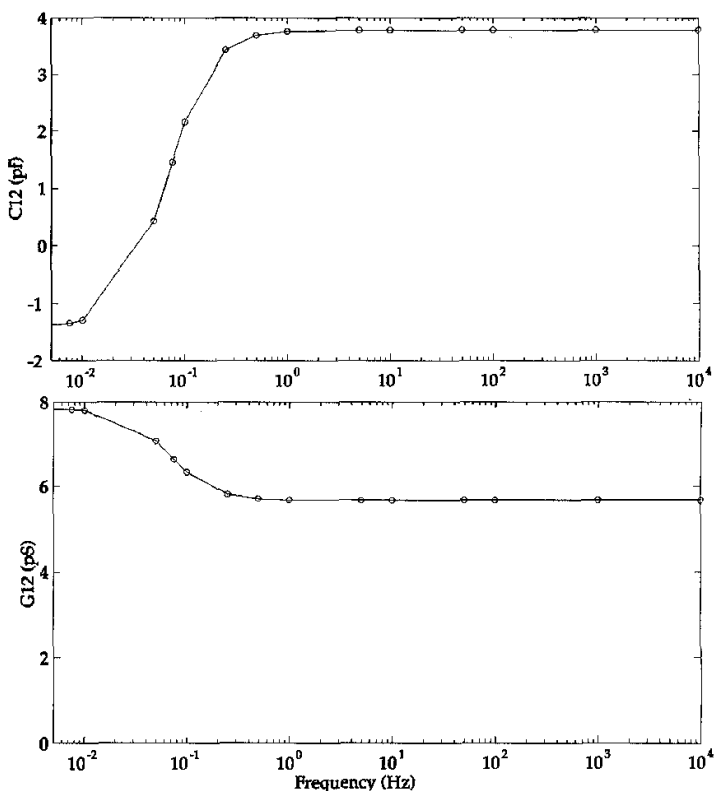

Figure 23. Simulation of the frequency dependent admittance matrix entries with finite-element software.

trodes along the $x$ axis. The effect of the former is alleviated by adding additional guard fingers on both sides of each wavelength, as shown in Figure 3.

Evaluation of the end effects along the $x$ axis can be accomplished in several ways. Closed-form solutions as well as empirical models of interdigital sensor discontinuities have been developed by several authors, for example [21]. At the same time, the precise geometry of the end areas usually differs from the idealized models. A simple experimental procedure was performed in order to approximate the equivalent capacitance of the end area. 


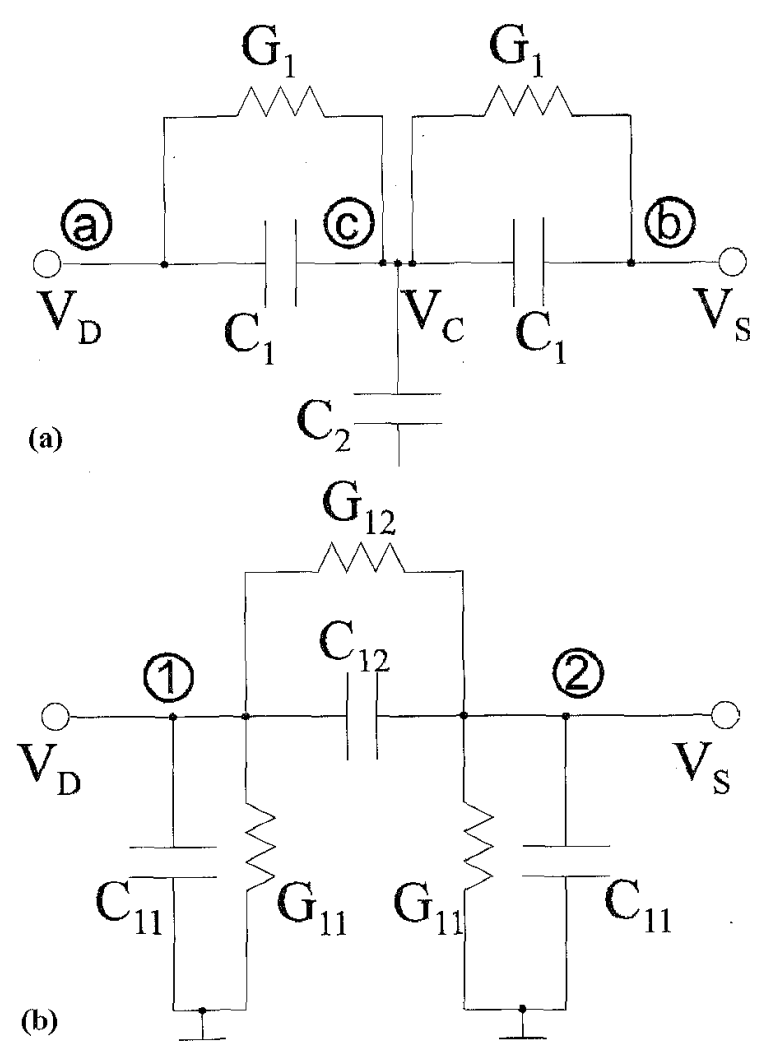

Figure 24. Justification of the negative transcapacitance values. (a) Equivalent circuit that approximately models sensor topology; (b) simple $\pi$ equivalent circuit with identical terminal characteristics.

Table 2. Values of load capacitances for the measurements reported in this paper.

\begin{tabular}{|l|r|r|r|r|r|r|}
\hline \hline \multicolumn{1}{|c|}{ Wavelength } & \multicolumn{2}{|c|}{$\lambda=1.0 \mathrm{~mm}$} & \multicolumn{2}{|c|}{$\lambda=2.5 \mathrm{~mm}$} & \multicolumn{2}{|c|}{$\lambda=5.0 \mathrm{~mm}$} \\
\hline Load capacitance, $\mathrm{pF}$ & Nominal & Measured & Nominal & Measured & Nominal & Measured \\
\hline Kapton sensor & 56.0 & 59.7 & 20.0 & 23.3 & 0 & 3.3 \\
Teflon sensor & 100.0 & 98.6 & 47.0 & 47.4 & 47.0 & 49.2 \\
\hline
\end{tabular}

The geometry of the end area of the $5 \mathrm{~mm}$ wavelength is shown in Figure 12. A piece of Lexan ${ }^{\mathrm{TM}}$ (GE polycarbonate) with measured permittivity $\varepsilon_{r}=3.17$ was placed in contact with the sensor so that the $y z$ plane (as defined by Figure 3 ) containing the air-Lexan boundary goes through $x=0$ in Figure 12. The Lexan sample was then repositioned in $0.25 \mathrm{~mm}$ increments in the direction of $x$, so that it gradually covers the electrode. Naturally, the equivalent capacitance $C_{12}$ increases as the Lexan moves along the $x$ axis. The change in capacitance during this measurement is shown in Figure 13. The growth becomes approximately linear when the Lexan slab position exceeds $x=3.125 \mathrm{~mm}$. The equivalent meander length of the end area can now be found by subtracting the output at the point $x=3.125 \mathrm{~mm}$ and at $x=0 \mathrm{~mm}$ and relating it to the linear function for $x>3.125 \mathrm{~mm}$. After taking the end effects into account and proper scaling of variables, the total meander lengths were empirically found to obey the relation $50+1.2 \lambda \mathrm{cm}$ for each wavelength, where $\lambda$ is expressed in $\mathrm{cm}$.

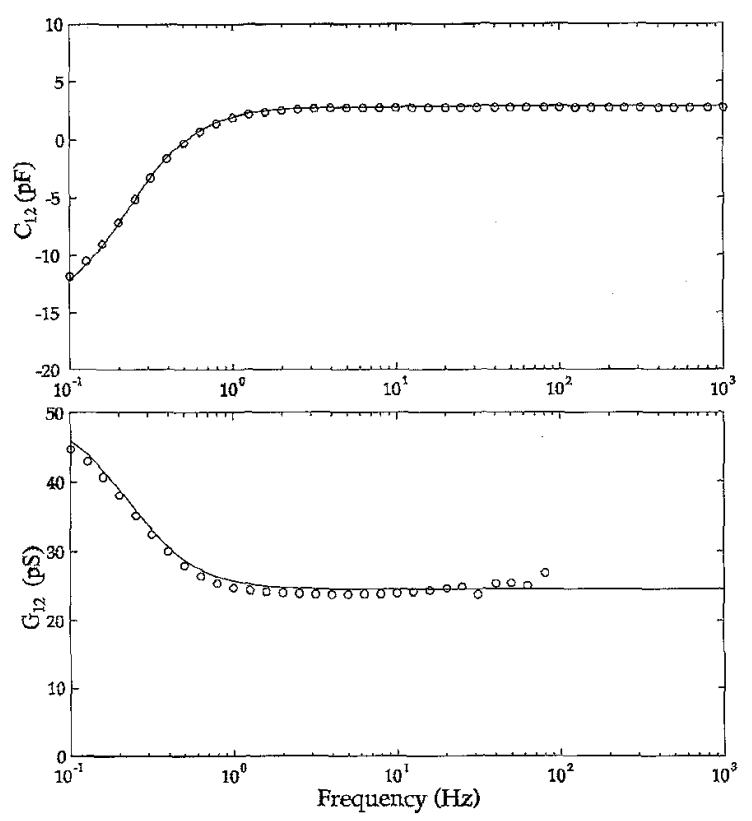

Figure 25. The measured and theoretical frequency response of the interelectrode capacitance $C_{12}$ and conductance $G_{12}$ for element values in Figure 24 of $G_{1}=9.8 \mathrm{G} \Omega, C_{1}=20 \mathrm{pF}$, and $C_{2}=100 \mathrm{pF}$.

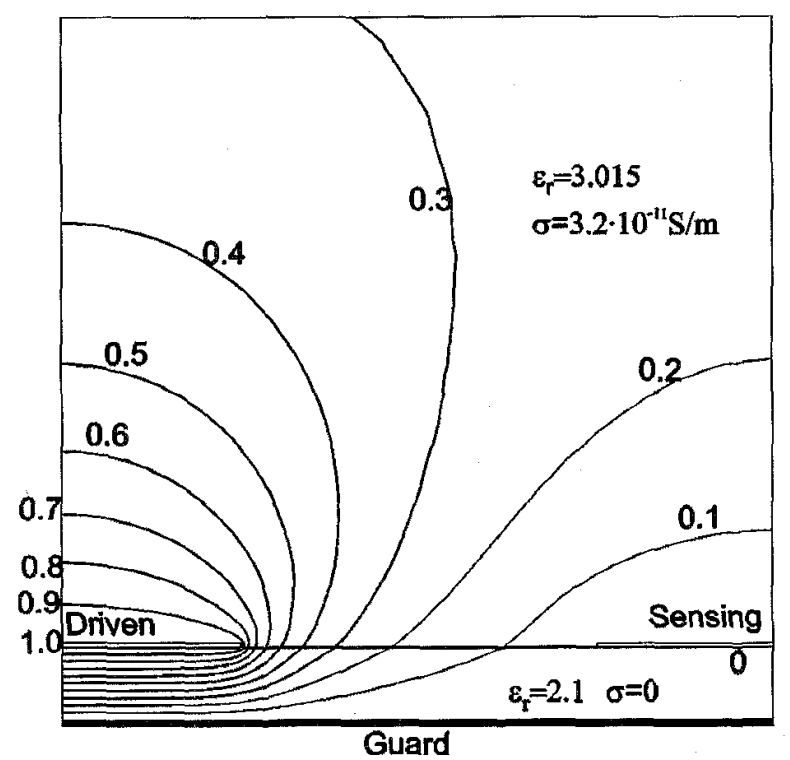

Figure 26. Equipotential lines for one half-wavelength of the $5.0 \mathrm{~mm}$ wavelength sensing electrode at $f=60 \mathrm{~Hz}$.

\subsubsection{CALCULATIONS WITH A FINITE-ELEMENT MODEL}

A continuum model of an interdigital sensor [2] provides a closedform solution for a set of idealized interdigitated electrodes (an infinite array of infinitely long microstrip conductors of zero thickness). Twodimensional finite-element modeling can incorporate a finite thickness of the electrodes and the continuous functional variation of material properties along all three coordinates.

Maxwell 2D, FE software for calculation of electric and magnetic fields and associated physical properties, has been used extensively 


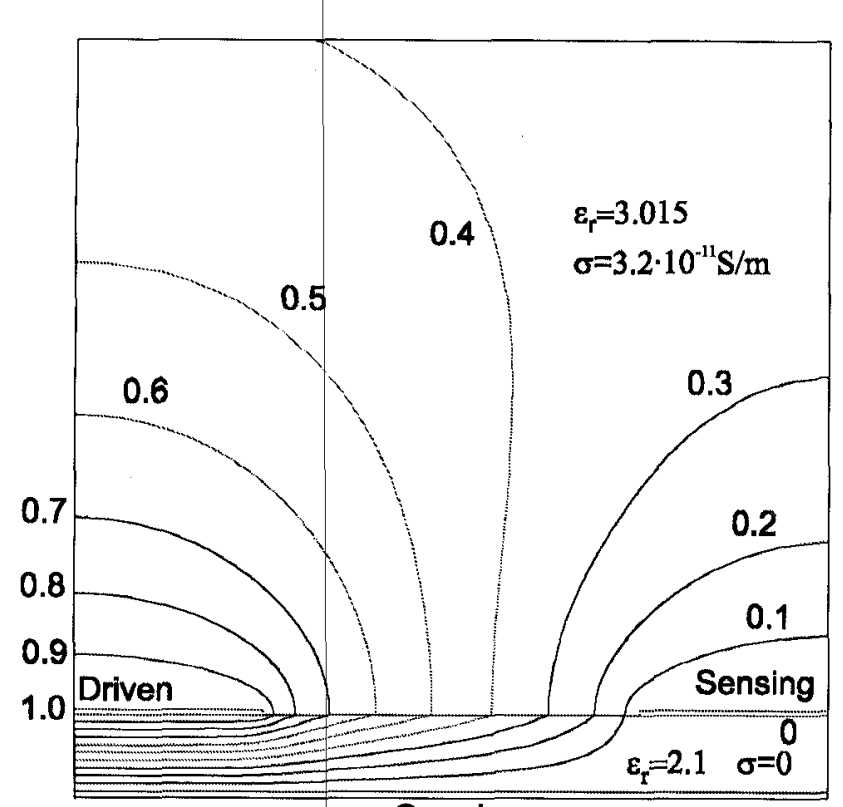

Guard

Figure 27. Equipotential lines for one half-wavelength of the $5.0 \mathrm{~mm}$ wavelength sensing electrode at $f=0.005 \mathrm{~Hz}$.

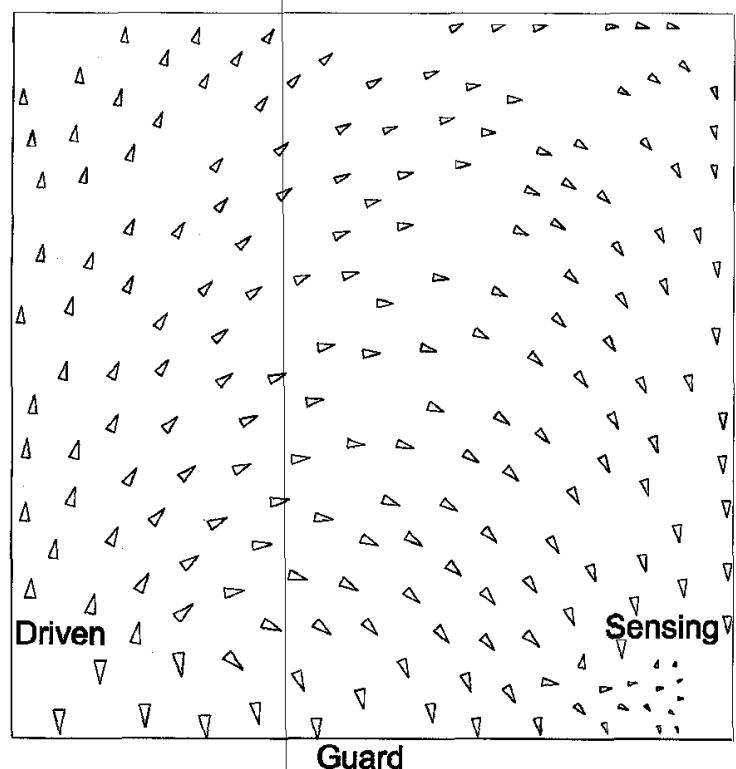

Figure 28. Distribution of the field in the space above the sensor at $f=60 \mathrm{~Hz}$. The size of the arrows is logarithmically proportional to the intensity of the electric field.

throughout the project. Analysis of electric field and equipotential line distributions provided important insight into the nature of approximations made. Calculation of the interelectrode capacitances provided benchmark comparisons between the theoretical calculations and measurements.

We return our attention to variation of the interelectrode capacitance with the width and thickness of the electrodes, and the ratio of the area covered with copper to the area of the exposed substrate. Curves in Figure 14 illustrate that the interelectrode capacitance varies considerably when the width of the electrodes changes. This information is useful

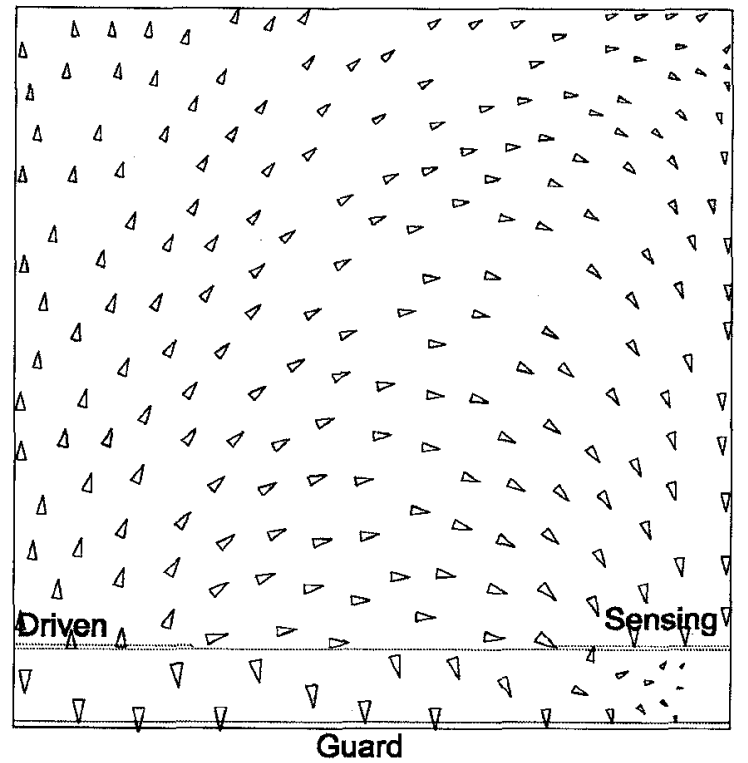

Figure 29. Distribution of the electric field in the space above the sensor at $f=0.005 \mathrm{~Hz}$. The size of the arrows is logarithmically proportional to the intensity of the electric field.

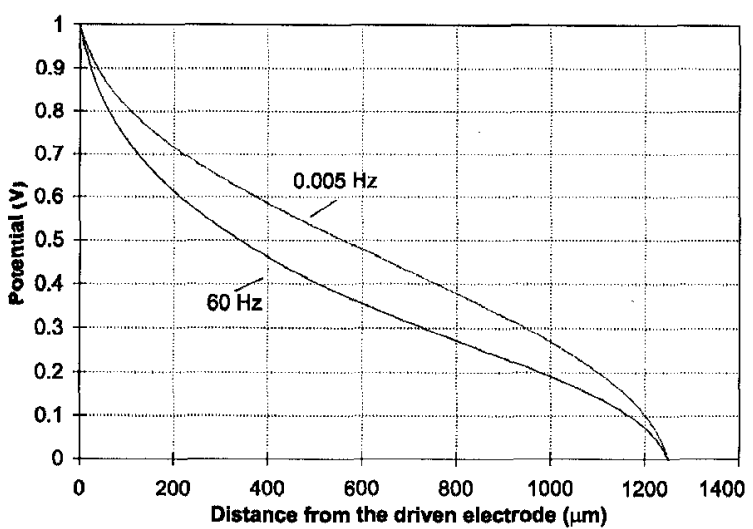

Figure 30. Distribution of the potential along the substrate-specimen interface between the driven and sensing electrodes.

Table 3. Calculated values of $C_{12}$ and $C_{11}(\mathrm{pF})$ per meter length for the Teflon sensor in air.

\begin{tabular}{|l|l|l|}
\hline wavelength & $C_{12}$ & $C_{11}$ \\
\hline$\lambda=1.0 \mathrm{~mm}$ & 7.14 & 16.1 \\
$\lambda=2.5 \mathrm{~mm}$ & 3.15 & 34.4 \\
$\lambda=5.0 \mathrm{~mm}$ & 2.03 & 60.2 \\
\hline
\end{tabular}

for two reasons. The resolution of the etching process on a flexible substrate is not always sufficient to ensure precise dimensions of the smaller wavelength, but even if this limitation is overcome, it may be useful to change the metalization ratio in order to extract additional information about the properties of the dielectric sample. The numerical values for the most frequently used case of the 50/50 metalization ratio computed with 'Maxwell' for the sensor in air are given in Table 3.

The thickness of the electrodes introduces less significant variation of the capacitance. Figures 15 and 16 show that the parasitic capacitance, defined here as the difference between the capacitance of the electrodes of finite height and the infinitely thin ones, is an approximately linear 
Table 4. Comparison of measured capacitance $C_{12}(\mathrm{pF})$ with pre-melted and re-hardened paraffin wax.

\begin{tabular}{|l|r|r|r|}
\hline Wavelength & pre-m & re-h & calc. \\
\hline$\lambda=1.0 \mathrm{~mm}$ & 3.63 & 6.36 & 6.29 \\
$\lambda=2.5 \mathrm{~mm}$ & 2.18 & 3.96 & 3.73 \\
$\lambda=5.0 \mathrm{~mm}$ & 1.32 & 2.38 & 2.35 \\
\hline
\end{tabular}

function of the thickness of the electrodes. This agrees with [7]. Additional analysis of the effects of the parasitic capacitance is given in the following section.

\section{EFFECTS OF SURFACE CONTACT QUALITY.}

\subsection{EXPERIMENTS}

A schematic view of a half-wavelength cross section of the sensor is shown in Figure 17. Several void regions can be identified. The cavity due to thickness of electrodes gives rise to a so-called parasitic capacitance [7]. In addition, some voids are formed above the electrodes, due to roughness and non-flatness of the electrodes and the tested material, as well as due to variations of the contact quality, which depends on the distribution of pressure above the specimen, which may be rigid or elastic.

A representative profilometer scan of the sensor's surface in Figure 18 shows that variation of the nominally $14 \mu \mathrm{m}$ electrode thickness is nearly negligible, on the order of $1 \mu \mathrm{m}$. The surfaces of the solid dielectric materials used in this study are even smoother for most cases. This leads us to conclude that the primary surface contact effect is due to non-flat surface deformations of the solid dielectrics and the sensor.

\subsubsection{PARAFFIN WAX}

The first set of measurements, serves to demonstrate the dramatic dependence of sensor output on the quality of contact with the specimen. Paraffin wax $\left(\varepsilon_{r} \approx 2.1, \sigma=0\right.$ at $1 \mathrm{kHz}$, melting temperature $57^{\circ} \mathrm{C}$ ) was first formed into a solid rectangular slab and placed directly contacting the sensor. Then, it was melted, poured over the sensor, and allowed to re-harden with paraffin wax filling the air cavities. After room temperature was reached and the paraffin wax solidified, measurement of interelectrode capacitance was made and compared to that of the premelted case. The results are summarized in Table 4 . The increase in $C_{12}$ after re-hardening from the melt is in good agreement with calculated values from a finite-element computer simulation which indicates that the paraffin wax has flowed into air cavities.

\subsubsection{HYZOD AND CORN OIL}

A similar experiment was conducted to further demonstrate the strong effect of air cavities and to explore the possibility of using matching permittivity liquids for high-precision measurements with irregularly shaped specimens. The approach to this measurement originates from a common technique of liquid displacement in dielectrometry measurements, for example [22]. In this technique, two materials, solid and liquid, whose permittivity values are very close and are used such that displacement of the liquid by the solid material does not change the response of the measurement circuit. Thus, the knowledge of properties of the liquid allows determination of the properties of the solid without making intricate electrodes.
Table 5. Measured values of $C_{12}(\mathrm{pF})$ with oil and Hyzod.

\begin{tabular}{|c|r|r|r|r|r|}
\hline \hline wavelength & step 1 & step 2 & step 3 & step 4 & simulation \\
\hline$\lambda=1.0 \mathrm{~mm}$ & 5.14 & 5.39 & 7.99 & 8.29 & 8.50 \\
$\lambda=2.5 \mathrm{~mm}$ & 3.96 & 3.96 & 5.25 & 5.61 & 5.59 \\
$\lambda=5.0 \mathrm{~mm}$ & 3.17 & 3.22 & 3.80 & 4.17 & 4.07 \\
\hline
\end{tabular}

Dielectric properties of all materials used in this paper were measured using parallel plate capacitive structures recommended in [22]. Liquid corn oil ( $\left.\varepsilon_{r}=3.015\right)$ and solid polycarbonate Hyzod ${ }^{\mathrm{TM}}\left(\varepsilon_{r}=\right.$ 3.00) were used in this experiment. Four consecutive steps of measurements at a frequency of $1 \mathrm{kHz}$ were taken:

1. Solid Hyzod is pressed against the sensor trapping a layer of air between the Hyzod and the sensor's surface.

2. Corn oil is then poured over the Hyzod, but since oil is quite viscous and air cavities are very small, the viscous oil did not fill in the cavities.

3. Hyzod specimen is then lifted so that oil can fill all cavities, after which the Hyzod is returned to its original position.

4. Hyzod is removed and measurements are taken with corn oil only

Table 5 shows that the interelectrode capacitance $C_{12}$ increases from a low to a high value as we go from step 1 to step 4 showing the importance of sample contact. The computer simulation assumes that the dielectric with relative permittivity $\varepsilon_{r}=3.015$ fills the entire space above the sensor, corresponding to step 4 .

It was observed that increase of the contact pressure and flexibility of the sensor does not lead to significant reduction of this effect. The quality of contact needs to be incorporated into the parameter estimation software relating measured gain and phase of $\widehat{G}$ to calculate permittivity and conductivity.

\subsection{CALCULATIONS}

Observations described in the previous Section lead to the need to distinguish between solid and liquid dielectrics when solving the inverse problem of material characterization from complex gain measurements. Calibration curves computed by finite-element simulation are compared to the experimental results in Figure 19 for various liquid and solid dielectrics.

Two lines correspond to two modeled cases: when the cavity between the electrodes is filled with dielectric ('liquid-simulation'), and when it is filled with air ('solid-simulation'). For all simulations the dielectric material and electrodes are assumed to be perfectly flat. The effect of the cavity becomes smaller as the wavelength increases. For the case $\lambda=5 \mathrm{~mm}$ it is hardly noticeable. At the same time, measurements show a large difference between solid and liquid for all wavelengths. Note that this difference far exceeds the effect of electrode height determined by simulation. This phenomenon is attributed to the air trapped above the electrodes (as was shown schematically in Figure 17).

The presumed equivalent air gap was simulated numerically (Figure 20). In this simulation, the material with $\varepsilon_{r}=2.1$ (Teflon, paraffin wax) is initially in ideal contact with the electrodes. The interelectrode capacitance decreases as the tested material moves away from the electrodes leaving behind the planar gap filled with air. In order to achieve a reduction of capacitance equal to that measured the equivalent gap has to be on the order of $40 \mu \mathrm{m}, \sim 3 \times$ the thickness of electrodes. 
The calibration program is being developed to include information about type of contact, surface roughness and surface deformation. One approach is to use redundant information from three independent measurements using the three-wavelength sensor to determine the equivalent planar air gap thickness.

\section{FULL FREQUENCY SWEEP \\ 5.1 EXPERIMENTAL RESULTS}

Gain and phase measurements with the capacitive divider are shown in Figure 21, and the results of conversion to the admittance matrix entries are in Figure 22 for corn oil. This measurement was done with the first generation of Teflon sensors (it has poorer etching tolerances than the second generation). In Figure 21, the gain is flat and phase is zero for high frequencies, which indicates a dominant capacitive behavior of the interelectrode admittance. The gain is zero for very low frequencies, which indicates a predominantly resistive behavior of the interelectrode admittance. To a first look, the overall gain-phase behavior is very reasonable for the system with constant coefficients, one pole and one zero.

The calculation of admittance matrix entries shows that this gainphase behavior corresponds to a system with frequency dependent coefficients, moreover, the capacitive part of the interelectrode admittance becomes negative at low frequencies. The values of the conductance slightly differ in the simulation and the measurements. This is attributed to a rise of conductivity during parallel plate measurements due to temperature variations.

\subsection{NEGATIVE TRANSCAPACITANCE}

It is appropriate to pause and discuss the calculated negative values of capacitance because it may not be immediately apparent to the reader how any lossy dielectric material can admit these values. Basic circuit theory can be used to help explain the measured negative capacitances. To understand the apparent negative capacitance in a more elementary setting consider the circuit shown in Figure 24(a) that approximately models the sensor topology in Figure 17. Suppose that we do not have the ability to measure the voltage at the node (C), and we are only interested in the circuit characteristics at nodes (a), (b), and ground. Then we can use the circuit shown in Figure 24(b) which exhibits identical behavior as the circuit shown in Figure 24(a) from the terminals of interest. The circuit parameters in Figure 24(b) are related to those of Figure 24(a) by

$$
\begin{aligned}
& G_{12}=2 G_{1} \frac{\omega^{2} C_{1}\left(C_{2}+C_{1}\right)+G_{1}^{2}}{\omega^{2}\left(2 C_{1}+C_{2}\right)^{2}+4 G_{1}^{2}} \\
& C_{12}=\frac{\omega^{2} C_{1}^{2}\left(2 C_{1}+C_{2}\right)+G_{1}^{2}\left(2 C_{1}-C_{2}\right)}{\omega^{2}\left(2 C_{1}+C_{2}\right)^{2}+4 G_{1}^{2}} \\
& G_{11}=G_{1}-2 G_{12}=\frac{\omega^{2} C_{2}^{2} G_{1}}{\omega^{2}\left(2 C_{1}+C_{2}\right)^{2}+4 G_{1}^{2}} \\
& C_{11}=C_{1}-2 C_{12}=\begin{array}{l}
\omega_{2}^{2} C_{1}\left(2 C_{1}+C_{2}\right)+2 G_{1}^{2} \\
C^{2}\left(2 C_{1}+C_{2}\right)^{2}+4 G_{1}^{2}
\end{array}
\end{aligned}
$$

Each parallel combination of $G_{1}$ and $C_{1}$ approximately represents the effective lossy layer of dielectric along the interface between driven and sensing electrodes, while $C_{2}$ represents the shunt capacitance through the substrate from interface to ground. Note that the capacitance $C_{12}$ is negative at low frequencies if $C_{2}>2 C_{1}$. The other three parameters $\left(G_{12}, G_{11}\right.$, and $\left.C_{11}\right)$ are positive definite for all frequencies.

We would like to emphasize a few points.

1. In theory, we can represent our distributed system well by a (possibly dense) network of resistors and capacitors which have a direct physical correspondence to the electrical properties of the material and sensor topology on an incremental scale. When we represent the same system by fewer elements while retaining the voltage/current terminal characteristics, the general equivalent circuit may include frequency dependent capacitors or resistors that can become negative over a range of frequency.

2. This only is possible in circuits with more than two terminals. In our case, we have the driving, sensing, and backplane electrodes. In a two-terminal circuit it is straightforward to prove (using Tellegen's theorem [8]) that any circuit containing only resistors and capacitors will appear from the terminals as a lossy capacitive system with no negative capacitors. As a consequence of this, in our circuits the driving point impedance seen by the source at the driven electrode will always be positively capacitive (and lossy).

3. Our elementary circuit example explains why the transcapacitances shown in Figures 22 and 23 have negative values at low frequencies but does not explain why the capacitance value is increasing at the very low frequencies in Figure 22. This occurs due to a separate phenomenon, the formation of an electrical double-layer capacitance [9]. Since 'Maxwell' software was not set up to account for such phenomena, the computer simulated capacitance does not increase at low frequencies but remains negative. An illustrative simulation of the frequency response agreed well with laboratory measurements as shown in Figure 25.

\subsection{FINITE ELEMENT COMPUTER SIMULATION}

Visualization of the electric scalar potential in the space above and inside the sensor for two limiting frequency cases is shown in Figures 26 and 27. The equipotential lines do not change significantly except for the region close to the Teflon-specimen interface, where different boundary conditions are imposed for the capacitive ( $\varepsilon E_{z}$ continuous) and the conductive ( $\varepsilon^{*} \widehat{E}_{z}$ continuous) case. Distribution of the electric field is shown in Figures 28 and 29.

The potential changes faster in the region directly adjacent to the conductors, as shown in Figure 30. A denser mesh of finite elements is formed in these regions by the FE software, and a denser distribution of the collocation points for the calculation of the electric potential is assumed in the continuum model [2].

\section{CONCLUSIONS}

number of improvements and modifications were necessary in order to achieve a successful implementation of $\omega-k$ dielectrometry. An improved version of a three-wavelength interdigital sensor was designed and tested in the laboratory setup. Different measurement 
schemes were explored, which led to extraction of the admittance matrix parameters bypassing the dependence on the material properties. Increasing testability and reliability of the measurements is achieved. While simulation of the geometry of the interdigital sensors can be done with high precision under reasonable assumptions, it does not necessarily lead to a full correspondence between theory and measurements.

It is demonstrated that cavities between the sensor and the test specimen can dramatically distort data employed for evaluation of material electrical properties. Discrimination of the quality of tested material contact to the sensor is necessary for correct evaluation of its electrical properties. Representative calibration curves for measurements for highly insulating homogenous materials are analyzed and recommendations are made towards further improvements of this technology.

\section{ACKNOWLEDGMENT}

Continuing support of the Electric Power Research Institute, under grant WO 8619-01, managed by Mr. S. Lindgren, and the National Science Foundation under grant No. ECS-9523128 is gratefully acknowledged. Especial thanks to Ansoft Corporation, for providing the finiteelement modeling software 'Maxwell'. The authors would like to thank other graduate students in the MIT High Voltage Research Laboratory, a division of the Laboratory for Electromagnetic and Electronic Systems, namely, Darrell Schlicker, Yanqing Du, and Yanko Sheiretov who have made important contributions to this study. Several experiments and simulations reported here were done with the help of visiting research engineer Jean Rivenc from Laboratoire de Genie Electrique, University of Toulouse, France, and MIT undergraduate students Boris Berdnikov and Binh Truong as part of the MIT Undergraduate Research Opportunities Program.

\section{REFERENCES}

[1] S. D. Senturia, N. F. Sheppard, Jr., H. L. Lee and D. R. Day, "In-situ Measurement of the Properties of Curing Systems with Microdielectrometry", J. Adhesion, Vol. 15, pp. 69-90, 1982.

[2] M. C. Zaretsky, L. Mouayad and J. R. Melcher, "Continuum Properties from Interdigital Electrode Dielectrometry", IEEE Transactions on Electrical Insulation, Vol. 23, No. 6, pp. 897-917, December 1988.

[3] M. C. Zaretsky, J. R. Melcher and C. M. Cooke, "Moisture Sensing in Transformer Oi] Using Thin-Film Microdielectrometry", IEEE Transactions on Electrical Insulation, Vol. 24, no. 6, pp. 1167-1176, Dec. 1989 .

[4] M. C. Zaretsky, P. Li and J. R. Melcher, "Estimation of Thickness, Complex Bulk Permittivity and Surface Conductivity Using Interdigital Dielectrometry", IEEE Transactions on Electrical Insulation, Vol. 24, no. 6, pp. 1159-1166, Dec. 1989

[5] M. C. Zaretsky and J. R. Melcher, "Complex Permittivity Measurements of Thin Films Using Microdielectrometry", 1986 Conference on Electrical Insulation and Dielectric Phenomena, Claymont, DE, pp. 462-471, Nov. 1986,

[6] A. Washabaugh, A. V. Mamishev, Y. Du and M. Zahn, "Dielectric Measurements of Semi-insulating Liquids and Solids", International Conference on Conduction and Breakdown in Dielectric Liquids, Rome, Italy, pp. 381-384, July 1996.
[7] P. A. von Guggenberg, Application of Interdigital Dielectrometry to Moisture and Double Layer Measurements in Transformer Insulation, PhD thesis, EECS Department, M. I. T., 1993.

[8] C. A. Desoer and E. S. Kuh, Basic Circuit Theory, Mc Graw Hill, New York, NY, p. 393, 1969.

[9] Y. K. Sheiretov and M. Zâhn, "Dielectrometry Measurements of Moisture Dynamics in Oil-Impregnated Pressboard", IEEE Transactions on Dielectrics and Electrical Insulation, Vol. 2, pp. 329-351, June 1995.

[10] P. A. von Guggenberg and J. R. Melcher, "A Three-Wavelength Flexible Sensor for Monitoring the Moisture Content of Transformer", Proceedings of the 3rd International Conference on Properties and Applications of Dielectric Materials, Vol. 2, pp. $1262-5,1991$.

[11] X. Du, M. Zahn, A. V. Mamishev and D. E. Schlicker, "Moisture Dynamic Measurements of Transformer Board Using A Three-Wavelength Dielectrometry Sensor", 1996 IEEE International Symposium on Electrical Insulation, Montreal, Quebec, Canada, pp. 53-56, June 16-19, 1996.

[12] Maxwell 2D Field Simulator, User's Reference, Ansoft Corporation, June 1994.

[13] M. C. Zaretsky, Parameter Estimation Using Microdielectrometry with Application to Transformer Monitoring, PhD thesis, Department of Electrical Engineering and Computer Science, Massachusetts Institute of Technoiogy, Cambridge, MA, November 1987.

[14] P. A. von Guggenberg and M. C. Zaretsky, "Estimation of One-Dimensional Complex-Permittivity Profiles: a Feasibility Study", Journal of Electrostatics, Vol. 34, no. 2-3, pp. 263-277, March 1995.

[15] A. K. Jonscher, Dielectric Relaxation in Solids, Chelsea Dielectrics Press, London, 1983.

[16] A. K. Jonscher, Universal Relaxation Law, Chelsea Dielectrics Press, London, 1996.

[17] A. V. Niamishev, Y. Du and M. Zahn, "Measurement of Dielectric Property Distributions Using Interdigital Dielectrometry Sensors", IEEE Conference on Electrical Insulation and Dielectric Phenomena, Virginia Beach, VA, pp. 309-312, October 1995.

[18] A. V. Mamishev and M. Zahn, "Techriques for Semi-Empirical Characterization of Material and Sensor Properties in Interdigital Dielectrometry", pp. 486-489, 1996 IEEE International Symposium on Electrical Insulation, Montreal, Canada.

[19] Y. K. Sheiretov and M. Zahn, "Dielectrometry Measurements of Moisture Dynamics in Oil-Impregnated Pressboard", 1994 IEEE International Conference on Properties and Applications of Dielectric Materials, University of Queensland, Brisbane, Australia, pp. 33-36, July 1994.

[20] D. E. Schlicker, Flow Electrification of Aged Transformer Oits, Master's thesis, EECS Department, M. I. T. 1996.

[21] M. Kirsching and R. H. Jansen, "Accurate, Wide Range Design Equations for the Frequency Dependent Characteristics of Parallel Coupled Microstrip Lines", IEEE Transactions on Microwave Theory Tech., Vol. MTT-32, pp. 83-90, January 1984.

[22] A-C Loss Characteristics and Permittivity (permittivity) of Solid Electrical Insulating Materials, Annual Book of ASTM Standards, D150-81.

[23] A. V. Mamishev, M. Zahn, B. C. Lesieutre and B. A. Berdnikov" "Influence of Geometric Parameters on Characteristics of an Interdigital Sensor", IEEE Conference on Electrical Insulation and Dielectric Phenomena, San Francisco, CA, pp. 550-553, October 1996.

This paper is based on a presentation given at the 12th International Conference on Conduction and Breakdown in Dielectric Liquids, Roma, Italy, 15-19 July 1996.

Manuscript was received on 25 March 1997, in revised form 9 February 1998. 